\title{
Pablo Marshall*
}

\section{The impact of quarantine on Covid-19 infections}

https://doi.org/10.1515/em-2020-0038

Received September 11, 2020; accepted February 13, 2021; published online March 10, 2021

\begin{abstract}
Objectives: Coronavirus has had profound effects on people's lives and the economy of many countries, generating controversy between the need to establish quarantines and other social distancing measures to protect people's health and the need to reactivate the economy. This study proposes and applies a modification of the SIR infection model to describe the evolution of coronavirus infections and to measure the effect of quarantine on the number of people infected.

Methods: Two hypotheses, not necessarily mutually exclusive, are proposed for the impact of quarantines. According to the first hypothesis, quarantine reduces the infection rate, delaying new infections over time without modifying the total number of people infected at the end of the wave. The second hypothesis establishes that quarantine reduces the population infected in the wave. The two hypotheses are tested with data for a sample of 10 districts in Santiago, Chile.

Results: The results of applying the methodology show that the proposed model describes well the evolution of infections at the district level. The data shows evidence in favor of the first hypothesis, quarantine reduces the infection rate; and not in favor of the second hypothesis, that quarantine reduces the population infected. Districts of higher socio-economic levels have a lower infection rate, and quarantine is more effective.

Conclusions: Quarantine, in most districts, does not reduce the total number of people infected in the wave; it only reduces the rate at which they are infected. The reduction in the infection rate avoids peaks that may collapse the health system.
\end{abstract}

Keywords: covid-19; epidemiology; impact of quarantine.

\section{Introduction}

Coronavirus has had a very significant impact on people's lives and the economy of many countries. By mid-July 2020, the number of people infected worldwide exceeded 15 million, and the number of deaths from Covid-19 exceeded 600 thousand. In many countries of the world, a controversy has arisen between the need to establish quarantines and other social distancing measures to protect people's health, and the need to return to economic activity to avoid sharper drops in occupation and the economy. Quarantine or confinement of people to their homes has been used in many countries to reduce and or delay infections, while the OECD (2020) estimates that each month of total quarantine in a country translates into a drop in GDP of up to 2 percentage points for this year 2020. Thus, a country that is quarantined for three months could see a decline of between 4 and 6 percentage points.

This study aims to measure the impact that quarantine has had in terms of the two objectives pursued by this measure: delaying and or preventing infections. On the one hand, quarantine produces fewer social contacts between people, which delays infections over time. This has been called "flattening the infection

The author is grateful to Aline Vineses for her work as a research assistant.

*Corresponding author: Pablo Marshall, Escuela de Administración, Pontificia Universidad Católica de Chile, Santiago, Chile, E-mail: pmarshall@uc.cl 
curve" to avoid very high peaks at certain points in time. This effect is important because it prevents the collapse of sanitary systems in terms of personnel, critical beds, and mechanical ventilators. The second purpose of quarantine is to reduce the population that acquires the virus in a wave of infections. In this case, quarantine could result in some people who were exposed to infection before quarantine being removed from this group by being confined to their homes. This second effect reduces the infected population without delaying the spread of infection over time. Measuring the magnitude of the two possible effects of quarantine is important for designing public policies that maintain an appropriate balance between the population's health and economic recovery. Measuring the impact of quarantine also allows designing actions in other countries, in future coronavirus waves, or in possible future epidemics similar to that of coronavirus.

In order to measure the quarantine effect, it is not possible to directly compare infection statistics in periods with and without quarantine. A correct analysis requires comparing the complete evolution of the wave of infections with and without quarantine.

Although there are many models in epidemiology that allow modeling infections in diseases such as those generated by coronavirus, most of them are based on extensions of the SIR model (susceptible - infected - recovered) originally proposed by Kermack and McKendrick (1927), which describes the evolution of infections in terms of quadratic differential equations. Extensions of the SIR model have also been used for modeling the spread of Covid-19 (Lofti et al. 2020).

This study uses a modification of the SIR model (Bass 1969; Bass et al. 1994) that has been extensively studied in marketing to represent the spread of infection to consumers when they purchase durable products. The advantage of the model proposed in this context is that it allows describing the infection process in relatively simple terms, the differential equation has an analytical solution and allows the impact of quarantine to be explicitly measured in terms of the two effects it can have on infections: delaying infections and/or reducing the infected population. This is the first application of the Bass model to model the evolution of diseases in a pandemic and to measure the impact of sanitary measures such as quarantine. In this sense, this study presents a methodological contribution.

The infection model proposed to describe the evolution of Covid-19 infections is applied to a sample of districts in Santiago, Chile, which has one of the highest numbers of infections per million inhabitants. The Chilean health authority has used a mixed strategy of periods with quarantine and periods without quarantine for different districts of the city of Santiago, which makes the reality of infections in Chile particularly appropriate to measure the impact of quarantine measures.

The studies that have been carried out to measure the impact of quarantine (Dandekar and Barbastathis 2020; Nussbaumer-Streit et al. 2020) show that the moment in which measures such as quarantine are established is very relevant to the impact of these measures. Measures are most effective when implemented early. In this sense, quarantine in Santiago, Chile, was established when the number of infected persons had been growing exponentially for several weeks.

The rest of this study is organized as follows: Section 2 describes different infection models and proposes the infection model that allows estimating the impact of quarantine in terms of delaying or flattening the infection curve and reducing the population susceptible to infection. Section 3 shows an application of the model to 10 districts of Santiago, and Section 4 concludes.

\section{Infection models and the impact of quarantine}

A traditional model in epidemiology to mathematically model the infectious spread of a disease is the SIR model (Kermack and McKendrick 1927), which establishes differential equations for the evolution of the population susceptible to acquiring the disease, of the infected population and of those who have recovered from the disease. Specifically, for the evolution of the infected population, the SIR model proposes that the infection rate depends linearly on the infected population. Additionally, the infected population does not remain in that state permanently because of the infected recover and stop infecting those who are susceptible. 
Since the development of the SIR model, many studies have been proposed to analyze and predict the evolution of infections from different diseases (Brauer et al. 2019). Most recent extensions of the SIR model consider additional population compartments in addition to the three initial categories mentioned (Ndairou et al.2020), models for different geographical regions (Brignano and Lavernaro 2020), and models for different demographic groups (Ellison 2020).

A constant dilemma in the formulation of infection models is that they are based on differential equations that are usually not integrable and, therefore, it is not possible to obtain an analytical solution to the evolution of infections. In some particular cases of the SIR model, closed analytical forms have been found, which facilitates the analysis, estimation, and validation of the model. The models with analytical solution correspond to the SIS model in which there is no immunity and the infected return to the susceptible state, and in the case of SIR when the susceptible and the infected go to the category of recovered with a constant recovery rate (Shabbir et al. 2010).

This study proposes a modification of the SIR model, which, although also based on differential equations, does have an analytical solution. The proposed model is simple and also allows incorporating control variables, such as the sanitary measures imposed by the authority in periods of infection in order to design and measure the impact of health policies. The model we propose is based on Bass (1969) and Bass et al. (1994) and has been extensively used in marketing to describe infection processes in the consumption of durable goods. The model can be described according to the differential equation

$$
\frac{\mathrm{d} F_{t}}{\mathrm{~d} t}=\left(p+q F_{t}\right) x_{t}\left(1-F_{t}\right)
$$

where $F_{t}$ is the proportion of the population infected until time $t, p$ and $q$ are parameters and the component $x_{t}$ depends on control variables. Equation (1) describes the behavior of the new infected at a time instant $t$. The right-hand side of Eq. (1) has three factors. The first parenthesis corresponds to the baseline infection rate that depends on parameters $p$ and $q$ and that grows linearly according to the number of persons infected. The larger the population infected, $F_{t}$, the higher the infection rate. The coefficient $p$ corresponds to the rate of first infections from outside the population under analysis, while $q$ is the infection rate within the population under analysis. The $x_{t}$ factor in (1) allows the model to dynamically change the infection rate according to control variables such as quarantine or social distancing. The third term on the right-hand side of (1) is the proportion of the population that has not been infected and is therefore exposed to the disease. The baseline model, which we call M1, assumes that $x_{t}=1$.

Model 1 is very similar to the SIR infection model. However, it has a simple analytical solution, allowing the incorporation of control variables into the factor $x_{t}$ and the initial infection rate $p$ appears explicitly in the model as a parameter to be estimated. In the specification (1), however, those who recover from the disease and therefore stop infecting are not subtracted from the infected as the SIR model does. If the proportion of the infected population is low, as is the case with Covid-19, in the current wave of infection, this effect is smaller. As we will see in the application of the model, empirically, there are no differences between the SIR model with explicit solutions (Shabbir et al. 2010) and the baseline model (1) with $x_{t}=1$. Recently, Lofti et al. (2020) predict the spread of the Covid-19 pandemic in Morocco using a model based on a variant of the SIR formulation similar to the model proposed in our study.

For the application of the model to coronavirus infections, we assume that the $x_{t}$ component has the form

$$
x_{t}=1-\beta C_{t}
$$

where $C_{t}$ is a dummy variable that takes values of 0 and 1 depending on whether or not the health authority has imposed a quarantine on the region analyzed, and $\beta$ is the parameter that measures the impact of quarantine in terms of reducing the infection rate. We expect this parameter $\beta$ to have a value greater than zero so that in quarantine periods the value of the $x_{t}$ factor is less than one, which decreases the infection rate, while in non-quarantine periods the component $x_{t}$ is equal to one. Equations (1) and (2) become the M2 model.

The component $F_{t}$ in (1) represents the cumulative proportion of people who have been infected. This proportion acts on the potentially infected population, which is not necessarily the total population of 
the region analyzed because some people isolate themselves, maintain social distance, and apply health protection measures in such a way that they are not part of the population exposed to infection. In models M1 and M2, the potentially infected population is fixed, and we represent it by $\alpha N$, where $N$ is the total population, and $\alpha$ is the proportion exposed to infection in the wave.

But if the potentially infected population varies with quarantine, we propose the specification

$$
N_{t}=\alpha N\left(1-\gamma C_{t}\right)
$$

where $\gamma$ is a coefficient that affects the potentially infected population according to the quarantine represented by the previously defined dummy variable $C_{t}$. A positive value is expected for the coefficient $\gamma$. The model (1) with $x_{t}=1$ and (3) is then our M3 model. In this model, quarantine reduces the population susceptible to infection but not the infection rate. Finally, a mixed model of the form (1)-(3) can be considered, where the effect of quarantine is two-fold. On the one hand, it reduces the infection rate and, on the other, it reduces the potentially infected population. We call this model M4.

In summary, the models considered that allow testing the hypotheses proposed for the impact of quarantine are as follows:

M1: baseline model without structural changes during the quarantine, $\beta=0$ and $\gamma=0$;

M2: model with decreased infection rate during the quarantine, $\beta>0$ and $\gamma=0$;

M3: model with the decreased infected population during the quarantine, $\beta=0$ and $\gamma>0$;

M4: model with lower infection rate and less infected population during the quarantine, $\beta>0$, and $\gamma>0$.

Model M1 is nested in all other models. Also, models M1, M2, and M3 are nested in M4, but M2 and M3 are not nested in each other. These two models correspond to the two hypotheses raised for the impact of quarantine.

Figure 1 shows the difference between the proposed models. The baseline model corresponds to model M1 without a quarantine effect in the light blue line. This model describes the evolution of infections before the quarantine. After the vertical line marking the beginning of quarantine in Figure 1, the model represents what would have happened if no quarantine had been applied. Model M2 corresponds to the one in which the infection rate decreases by $30 \%$. The infection curve is attenuated and shifts to the right, lowering the peak of infections. The potentially infected population, however, remains constant so that the decrease of infections in the peak is offset by a longer duration of the infection period. In other words, the curve is flattened. Model M3 does not change the infection rate, but it does change the number of infections. In the figure, this population decreases by $30 \%$. The infection curve does not shift but rather decreases. Naturally, it is possible that in practice, a combined scenario may occur in which both hypotheses coexist.

The two hypotheses proposed have very different consequences in terms of public policies. M2 model does not affect the total number of people who become infected. It only affects the time in which the infection occurs. This makes it possible to manage the resources of medical centers so that there is no over-demand

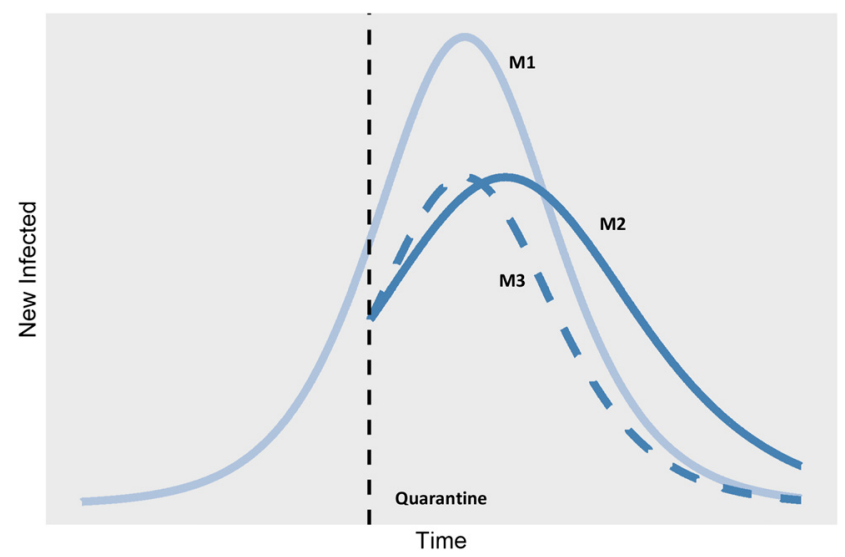

Figure 1: Quarantine impact models. Evolution of infections before and after quarantine according to different versions of the model. Model M1 assumes that there is no impact of quarantine on infections. Model M2 assumes that the quarantine reduces the infection rate while model M3 assumes that the quarantine reduces the infected population in the wave. 
for resources at any given point in time. M3 model, on the other hand, reduces the infected population and, therefore, the number of people who will be seriously affected by the virus. The peak of infections is similar in both models, as shown in Figure 1.

\section{Application of the model to districts in Santiago, Chile}

Chile is one of the countries with the highest number of Covid-19 infections per million inhabitants in the world. The health authority has used a mixed strategy of periods with and without quarantine for different districts in the city of Santiago. This means that in a period of 22 weeks, from the beginning of March to mid-July, several districts of Santiago have had both quarantine and non-quarantine periods. Throughout this time span, there has been a very significant wave of infections.

For the application of the model presented in the previous section, a sample of 10 districts of Santiago with different socio-economic levels, different population sizes, and different mobility rates has been selected in order to analyze possible differences across districts (Ministry of Science 2020). One criterion for the selection of districts is that they must have a sufficiently long period with and without quarantine in order to be able to estimate the impact of this measure. The districts considered in the sample have between eight and 12 weeks of quarantine and the remaining weeks without quarantine. Table 1 shows demographic characteristics of

Table 1: Characteristics of selected districts.

\begin{tabular}{lcl}
\hline District & Population (thousands) & Socio-economic level \\
\hline La Florida & 402 & Medium \\
La Pintana & 189 & Low \\
La Reina & 100 & High \\
Las Condes & 331 & High \\
Maipú & 579 & Medium \\
Peñalolén & 267 & Medium \\
Providencia & 158 & High \\
Pudahuel & 253 & Low \\
Quilicura & 255 & Low \\
San Bernardo & 335 & Low \\
\hline
\end{tabular}

The population of each district in thousands of people, according to the 2017 Census. Socio-economic level according to the percentage of households below the poverty line in 2017.

Table 2: Estimated impact of quarantine on districts of Santiago, Chile.

\begin{tabular}{|c|c|c|c|c|c|c|c|}
\hline District & $p$ & $q$ & $\alpha$ & $\beta$ & $\gamma$ & $R^{2}$ & Error, \% \\
\hline La Florida & $0.275 \times 10^{-4}$ & $0.716^{* * *}$ & $0.033^{* * *}$ & $0.273^{* * *}$ & & 0.993 & $13.3 \%$ \\
\hline La Pintana & $0.900 \times 10^{-4 * * *}$ & $0.600^{* * *}$ & $0.050^{* * *}$ & & & 0.995 & $11.7 \%$ \\
\hline La Reina & $0.200 \times 10^{-4 * * *}$ & $0.715^{* * *}$ & $0.021^{* * *}$ & $0.340^{* * *}$ & & 0.997 & $8.2 \%$ \\
\hline Las Condes & $1.111 \times 10^{-4 * *}$ & $0.647^{* * *}$ & $0.017^{* * *}$ & $0.276^{* * *}$ & & 0.987 & $17.4 \%$ \\
\hline Maipú & $0.677 \times 10^{-4 * * * *}$ & $0.605^{* * *}$ & $0.024^{* * *}$ & $0.152^{* * *}$ & & 0.995 & $11.1 \%$ \\
\hline Peñalolén & $0.075 \times 10^{-4}$ & $0.847^{* * *}$ & $0.042^{* * *}$ & $0.293^{* * *}$ & & 0.988 & $17.8 \%$ \\
\hline Providencia & $1.300 \times 10^{-4}$ & $0.612^{* * *}$ & $0.019^{* * *}$ & $0.214^{* *}$ & & 0.968 & $27.4 \%$ \\
\hline Pudahuel & $0.402 \times 10^{-4 *}$ & $0.666^{* * *}$ & $0.037^{* * *}$ & $0.194^{* * *}$ & & 0.993 & $13.8 \%$ \\
\hline Quilicura & $0.719 \times 10^{-4 * * *}$ & $0.614^{* * *}$ & $0.051^{* * *}$ & & $0.411^{* * *}$ & 0.997 & $8.3 \%$ \\
\hline San Bernardo & $1.836 \times 10^{-4 * * * *}$ & $0.515^{* * *}$ & $0.033^{* * *}$ & & & 0.996 & $10.2 \%$ \\
\hline
\end{tabular}

Estimation of models in each district, $R^{2}$ statistic, and forecasting error in the sample. All models contain 22 observations. The statistical significance of coefficients is denoted by ${ }^{*}(10 \%),{ }^{* *}(5 \%)$ and ${ }^{* * *}(1 \%)$. 
the districts in the sample: population in thousands of people and socio-economic levels according to the proportion of households below the poverty line. For each of the districts in the sample, the analytical solution of the differential Eq. (1) was estimated by non-linear least squares incorporating a random error and the two hypotheses about the impact of quarantine defined in (2) and (3). In estimating the coefficients, the parameters $\beta$ and $\gamma$ were constrained to be greater than or equal to zero. The results of the estimation are shown in Table 2. The estimation strategy was to estimate the more general model, M4, and then reduce the model according

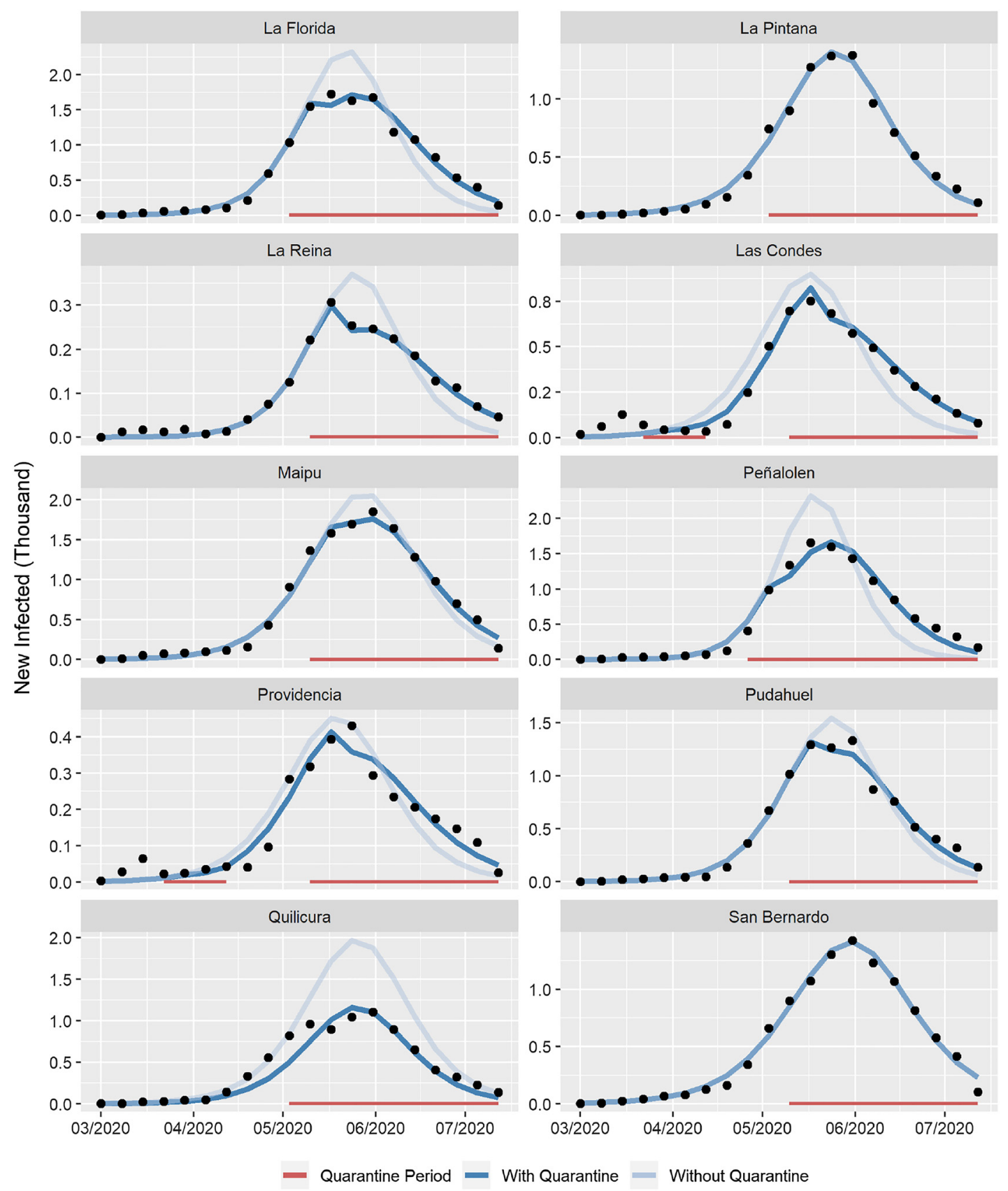

Figure 2: Adjusted model with quarantine and model without quarantine by district.

Models adjusted for the different districts. Black dots show infection observations in each week, the blue line corresponds to the newly infected trajectory according to the estimated model, while the lighter line describes the evolution that new infections of each week would have had if no quarantine had been established. Quarantine periods are marked in red. 
to non-significant or equal to zero coefficients. The only coefficient that was kept in the model, regardless of its significance, was the parameter $p$ because it corresponds to the rate of first infections from outside the district. If this parameter equals zero, no infections occur.

The results of Table 2 show that the values of the parameter $p$ are very small, but they are still significant in most of the districts. The infection rate $q$ is always significant and very stable across districts. The proportion of the total infected population in each district, $\alpha$, is small and always significant. A negative and strong correlation is observed between $\alpha$ and the socio-economic level (-0.77). In other words, districts with higher socio-economic level have a lower proportion of the infected population. On the other hand, for most of the selected districts, the hypothesis $\beta=0$ is rejected, which is consistent with the hypothesis that quarantine delays infections. The average $\beta$ coefficient is $24.9 \%$. It is observed that the reduction of the infection rate by quarantine, $\beta$, has a positive and strong correlation (0.79) with the socio-economic level. That is, in districts of higher socio-economic level, quarantine is more effective in delaying infections. In other words, the hypothesis $\gamma=0$ is accepted in all but one of the districts. This means that there is no evidence in the data that quarantine reduces the population that is infected in a wave. There are two low-socio-economic-level districts for which the hypothesis $\beta=\gamma=0$ is accepted.

The average error level when predicting new infections in a week is $13.9 \%$, and the average error when predicting cumulative infections in the wave is $1.6 \%$. Elmousalami and Hassanien (2020) present a comparison of forecasting models on COVID-19 infections, at the aggregated level in the world, using time series models and mathematical formulation. The mean absolute percentage error of their predictions fluctuates, across methodologies, between 10 and 20\%. The proposed model in our study, Eqs. (1)-(3), compares favorably with these prediction errors. In addition, the average $R^{2}$ indicator of the models is 0.991 , which shows that the model represents well the evolution of infections in the sample period.

In estimating the different models, we observe a moderate degree of correlation between the estimated $\beta$ and $\gamma$ coefficients. In one of the districts-Maipú -the estimation of the complete model accepts the hypothesis $\beta=\gamma=0$, but when estimating models M2 and M3, which contain only one of these parameters, both coefficients came out significant. Since M2 and M3 are not nested, model M2 was preferred because the likelihood ratio of M2 relative to M3 was equal to 3.0. In a Bayesian context, this means that model M2, relative to model M3, has a $75 \%$ probability.

In order to compare the estimates of the proposed model with the traditional SIR model, the M1 baseline model and the analytical versions of the SIR model were estimated without the quarantine variable (Shabbir et al. 2010). In all districts, the estimates are almost exactly the same in terms of the likelihood function and in terms of the infection rate $q$.

Figure 2 shows the models adjusted for the selected districts. Black dots correspond to the infection observations in each week; the blue line shows the trajectory of new infected according to the estimated model, while the lighter line corresponds to the evolution that new infections would have had each week if no quarantine had been established. Quarantine periods are marked in red. The difference between the peaks of the light blue and blue curves corresponds to the reduction of infections achieved in the peak week with the highest number of infections.

\section{Conclusions}

Coronavirus has had a profound effect on people's lives and on the economy of many countries, which has generated controversy between the need to establish quarantines and other social distancing measures to protect people's health, and the need to restart the economy.

This study measures the impact of quarantine in terms of the two objectives pursued by this measure: to delay and/or prevent infections. This measurement is important for designing public policies that ensure an appropriate balance between the population's health and economic reactivation. In order to measure the two potential effects of quarantine, we propose and apply a modification of the SIR model that has several 
advantages: it is simple, has an analytical solution, and allows the incorporation of control variables for measuring the impact of health authority policies during the wave of infections.

The model is applied to 10 districts of Santiago, Chile, which during 22 weeks, have been affected by periods with and without quarantine. The results show that the proposed Bass model adjusts very well to the data. The average prediction error across districts is $13.9 \%$, which compares favorably with other studies that predict infections for the Covid-19 (Elmousalami and Hassanien 2020). From a public health perspective, the impact of quarantine is to significantly reduce the infection rate, preventing peaks that could collapse the health system. The average reduction in the infection rate is $24.9 \%$. Furthermore, it is observed that the reduction in the infection rate has a strong positive correlation (0.79) with the socio-economic level. The hypothesis that quarantine periods reduce the population exposed to infection in a wave is rejected in nine of the 10 districts analyzed. This result is probably explained by the fact that many people have established their own social distancing and personal health care measures, irrespective of the quarantines decreed by the authority. For these persons, the probability of contracting the disease remains very low in periods with and without quarantine. Another hypothesis that explains these results is that quarantines were decreed too late when infections already showed exponential growth. Studies carried out to measure the impact of quarantine (Dandekar and Barbastathis 2020; Nussbaumer-Streit et al. 2020) show that quarantines are more effective in reducing infections if implemented early.

Research funding: None declared.

Author contribution: All authors have accepted responsibility for the entire content of this manuscript and approved its submission.

Competing interests: Authors state no conflict of interest.

Informed consent: Not applicable.

Ethical approval: Not applicable.

\section{References}

Bass, F. 1969. “A New Product Growth for Model Consumer Durables.” Management Science 15 (5): $215-27$.

Bass, F., T. V. Krishnan, and D. C. Jain. 1994. "Why the Bass Model Fits without Decision Variables.” Marketing Science 13 (2): 203-23.

Brauer, F., C. Z. Castillo-Chavez, and Z. Feng. 2019. Mathematical Models in Epidemiology. New York: Springer-Verlag.

Brignano, L., and F. Lavernaro. 2020. “A Multi-Region Variant of the SIR Model and its Extensions.” arXiv:2003.09875 [q-bio.PE].

Dandekar, R., and G. Barbastathis. 2020. "Quantifying the Effect of Quarantine Control in Covid-19 Infectious Spread Using Machine Learning.” Preprint Medxriv.

Ellison, G. 2020. “Implications of Heterogeneous SIR Models for Analyses of COVID-19.” NBER Working Paper No. 27373JEL No. I18.

Elmousalami, H. H., and A. E. Hassanien. 2020. "Day Level Forecasting for Coronavirus Disease (Covid-19) Spread: Analysis, Modeling and Recommendations.” arXiv:2003.07778.

Kermack, W. O., and A. G. McKendrick. 1927. "Contribution to the Mathematical Theory of Epidemics.” Proceedings of the Royal Society of London 115: 700-21.

Lotfi, B., I. Lotfi, and O. Aoun. 2020. "Modeling the Spread of Covid-19 Pandemic: Case of Morocco.” Epidemiologic Methods 9 (S1): 1-10.

Ministry of Science, 2020. www.minciencia.gob.cl/covid19.

Ndairou, F., I. Area, J. J. Nieto, and D. F. M. Torres. 2020. "Mathematical Modeling of Covid-19 Transmission Dynamics with a Case Study of Wuhan.” Chaos, Solitons \& Fractals 135: 1-6.

Nussbaumer-Streit, B., V. Mayr, A. I. Dobrescu, A. Chapman, E. Persad, I. Klerings, G. Wagner, U. Siebert, C. Christof, C. Zachariah, and G. Gartlehner. 2020. "Quarantine Alone or in Combination with Other Public Health Measures to Control COVID-19: A Rapid Review.” Cochrane Database of Systematic Reviews 4: CD013574.

OECD. 2020. "Evaluating the Initial Impact of COVID-19 Containment Measures on Economic Activity.” www.oecd.org. June.

Shabbir, G., H. Khan, and M. A. Sadiq. 2010. “An Exact Solution of a Particular Case of SIR and SIS Epidemic Models.” arXiv:1012.5035. 